Nature. 2014 January 16; 505(7483): 335-343. doi:10.1038/nature12985.

\title{
Mitochondrial form and function
}

\author{
Jonathan R. Friedman ${ }^{1}$ and Jodi Nunnari ${ }^{1}$ \\ ${ }^{1}$ Department of Molecular and Cellular Biology, College of Biological Sciences, University of \\ California, Davis, California 95616, USA
}

\begin{abstract}
Mitochondria are one of the major ancient endomembrane systems in eukaryotic cells. Owing to their ability to produce ATP through respiration, they became a driving force in evolution. As an essential step in the process of eukaryotic evolution, the size of the mitochondrial chromosome was drastically reduced, and the behaviour of mitochondria within eukaryotic cells radically changed. Recent advances have revealed how the organelle's behaviour has evolved to allow the accurate transmission of its genome and to become responsive to the needs of the cell and its own dysfunction.
\end{abstract}

Mitochondria arose around two billion years ago from the engulfment of an aproteobacterium by a precursor of the modern eukaryotic cell ${ }^{1}$. Although mitochondria have maintained the double membrane character of their ancestors and the core of ATP production, their overall form and composition have been drastically altered, and they have acquired myriad additional functions within the cell. As part of the process of acquiring new functions during evolution, most of the genomic material of the a-proteobacterium progenitor was rapidly lost or transferred to the nuclear genome ${ }^{2}$. What remains in human cells is a small, approximately 16 kilobase, circular genome, which is present in cells in a vast excess of copies relative to nuclear chromosomes.

The human mitochondrial genome contains genetic coding information for 13 proteins, which are core constituents of the mitochondrial respiratory complexes I-IV that are embedded in the inner membrane. Functioning together with the Krebs' cycle in the matrix, the respiratory chain creates an electrochemical gradient through the coupled transfer of electrons to oxygen and the transport of protons from the matrix across the inner membrane into the intermembrane space. The electrochemical gradient powers the terminal complex $\mathrm{V}$ of the chain, ATP synthase, which is an ancient rotary turbine machine that catalyses the synthesis of most cellular ATP. The electrochemical potential is harnessed for additional crucial mitochondrial functions, such as buffering the signalling ion $\mathrm{Ca}^{2+}$ through uptake by a uniporter in the inner membrane ${ }^{3,4}$. A reduction in the electrochemical potential of mitochondria in cells has evolved as a read-out for mitochondrial functional status, which, as

(C) 2014 Macmillan Publishers Limited. All rights reserved Correspondence should be addressed to J.N. (jmnunnari@ucdavis.edu).

The authors declare no competing financial interests. 
discussed later, creates signals to activate pathways that repair and/or eliminate defective mitochondria.

We know from a combination of proteomics, genomics and bioinformatics that modern day mitochondria are comprised of well over 1,000 proteins; the composition is plastic in nature, varying with and between species in response to cellular and tissue-specific organismal needs ${ }^{5-7}$. The origin of the mitochondrial proteome is a mixture of 'old' bacterial and 'new' eukaryotic-derived proteins ${ }^{2}$. For example, the mitochondrial DNA (mtDNA) replication and transcription machines have distinct evolutionary origins in bacteriophage ${ }^{8-10}$, whereas the mitochondrial translational machinery has a clear evolutionary relationship to bacteria ${ }^{11}$. In addition to protein components, the mitochondrial genome encodes 22 transfer RNAs and 2 mitochondrial ribosome-coding RNAs, which are essential components of its own translational apparatus. Mitochondrial ribosome assembly in the mitochondrial matrix is a relatively complex and highly regulated process, which involves mitochondrial ribosomecoding RNA processing and maturation and the assembly of mitochondrial ribosomal proteins into small and large subunits ${ }^{12}$. However, only a fraction of mitochondrial ribosome proteins have identifiable homologues in bacteria ${ }^{13}$. The roles of mitochondrial specific ribosomal proteins are not understood, but these proteins are thought to have evolved to regulate the coordination of mitochondrial translation with extra-mitochondrial pathways in eukaryotic cells. Thus, like many mitochondrial machines, the ribosome is a mix of old and new innovations.

The nucleus-encoded proteins that make up most of the mitochondrial proteome are translated on cytosolic ribosomes and actively imported and sorted into mitochondrial subcompartments by outer and inner membrane translocase machines in a manner that is dependent on the electrochemical potential ${ }^{14,15}$. Transcriptional, posttranscriptional and post-translational modes of regulation exist for nucleus-encoded mitochondrial proteins. In humans, transcriptional regulation of mitochondrial biogenesis occurs through the action of the PGC-1 family of co-activators, which respond to changes in nutrient status, such as $\mathrm{NAD}^{+} / \mathrm{NADH}$ and AMP/ATP ratios (sensed through SIRT1 and AMPK, respectively), as well as environmental signals ${ }^{16,17}$. Combinatorial interactions between PGC-1 co-activators and specific transcription factors (NRF1, NRF2 and ERR) balance and specify the major functional pathways within mitochondria. Through the induction of nuclear genes that directly impinge on the maintenance of mtDNA, these interactions coordinate the nuclear and mitochondrial genomes ${ }^{18}$. Evidence in yeast suggests that nuclear-transcribed messenger RNAs encoding mitochondrial proteins are post-transcriptionally localized to the mitochondrial outer membrane in a highly regulated spatial and temporal manner, and coordinately translated ${ }^{19,20}$. Although the underlying molecular mechanisms of mRNA targeting to mitochondria are poorly understood, such pathways will probably be important in polarized cells such as neurons. Post-translational modifications, such as phosphorylation of mitochondrial import machinery components by cytosolic kinases, ultimately fine-tunes the proteome in response to metabolic cues ${ }^{21}$.

Mutations in either mtDNA genes or nuclear genes that encode the mitochondrial proteins required for aerobic ATP production cause a diverse and often devastating array of human mitochondrial diseases, which can affect any organ in the body at any point during a 
person's life ${ }^{22}$. In addition, there is a high degree of clinical heterogeneity in mitochondrial diseases. Some of his heterogeneity can be explained by the fact that human cells can contain a variable ratio of mutated and wild-type mtDNA, a state called heteroplasmy. This seems to be the case for mtDNA mutations in protein-coding regions of the mitochondrial genome, in which an increase in mutant load gives rise to more severe disease phenotypes. However, disease heterogeneity that cannot be explained by heteroplasmy also exists when mutations are present in non-coding mitochondrial tRNAs. In addition, mutations in genes with a shared function, such as genes encoding subunits of complex I of the respiratory chain, lead to disease manifestations that are vastly different, such as optic nerve atrophy in adults or encephalopathy in infants ${ }^{23,24}$. Recent studies also point to a causal link between mutations in mtDNA and ageing, probably resulting from mtDNA-linked defects in somatic stem cells ${ }^{25,26}$.

The role of mitochondria in disease has been expanded beyond the respiratory chain, as defects in additional mitochondrial functions and behaviours have been linked to cancer, metabolic disorders and neurodegenerative diseases, such as Alzheimer's, Parkinson's and Huntington's disease ${ }^{22}$. In general, however, our current understanding of the underlying relationship between mitochondrial phenotype and disease is poor and requires a better understanding of mitochondrial organization, as well as the connections mitochondria have with the nuclear genome and extra-mitochondrial pathways in different cell types and at the organismal level. To address this deficit, a renaissance in mitochondrial research has emerged, hastened by recent advances in genetics, systems-based approaches and our ability to visualize mitochondria at high temporal and spatial resolution. In this Review, we discuss how this renaissance is both redefining and extending our knowledge about mitochondrial behaviour and communication.

\section{The mitochondrial chromosome}

Given the importance of mtDNA-encoded genes for mitochondrial function, it is not surprising that there are dedicated mechanisms that actively control the structure and distribution of mitochondria and mtDNA, but in higher eukaryotes, these mechanisms are divergent from those of their ancestors ${ }^{22}$. Unlike bacteria, in most cell types, individual mitochondria do not exist; instead, they comprise a connected network containing multiple copies of the mitochondrial chromosome, forming a 'syncytium' (Fig. 1a, b). Like bacterial and nuclear chromosomes, mtDNA is highly compacted within the mitochondrial matrix, and consequently mtDNA-protein complexes can be visualized as punctate structures, termed nucleoids, within networks. The mechanism underlying mtDNA condensation was illuminated by researchers who solved the crystal structure of the most abundant mtDNAassociated protein in mammalian cells, mitochondrial transcription factor A (TFAM). The structure indicates that TFAM both binds and bends short stretches of mtDNA, forming loops that allow mtDNA packaging ${ }^{27,28}$. TFAM also plays a crucial part in mtDNA transcription, and its expression controls mtDNA copy number in cells, making it a central player in mtDNA maintenance and transmission ${ }^{29,30}$.

Additional proteins that are crucial for mtDNA maintenance are localized at nucleoids. These proteins include replication and repair machinery, which, in humans, includes DNA 
polymerase $\gamma$ and its accessory proteins, such as the replication helicase twinkle ${ }^{31}$.

Mutations in genes encoding these and additional factors required for mtDNA maintenance cause a spectrum of human mitochondrial diseases, but at the molecular level, it is still not understood how these and the many other identified mtDNA-associated proteins are assembled together and organized to build nucleoids ${ }^{32}$. This is despite the fact that proteomic inventories of mtDNA-associated proteins have been determined for many species $^{33-35}$. In addition, both higher order nucleoid organization and the mode of mtDNA replication are variable between biological kingdoms, resulting in further complexity. These differences are a consequence of the composition of the genome and the nucleoid. For example, yeast possesses an active recombination machine similar to Rad52 recombination proteins, and replication is probably primarily recombination driven ${ }^{36}$. As a result, relative to the human mitochondrial genome, the larger 80 kilobase yeast genome is packaged in multiple copies in the nucleoid. By contrast, the mechanism of human mtDNA replication is recombination independent in most tissues and occurs through strand displacement ${ }^{37,38}$. Super-resolution imaging consistently indicates that human nucleoids contain a relatively small number of mtDNA molecules and thus are more solitary in nature ${ }^{39,40}$. These differences in organization and transmission modes of the mitochondrial chromosome greatly impact the segregation behaviour of mtDNA.

\section{Modes of mtDNA segregation}

The multi-copy nature of mtDNA means the mode of mtDNA transmission is viewed as stochastic or 'relaxed' in most cell types and thus is radically different from that of nuclear genes $^{41}$. On an organismal level, in humans for example, mtDNA is inherited in a uniparental maternal manner, and paternal mtDNA is actively destroyed after fertilization $^{42,43}$. Mitochondria in egg and sperm also have different functional states, shapes and cellular distributions, and these differences are probably important to confer fitness. In addition, from generation to generation mtDNA genotypes rapidly segregate, indicating that a 'bottleneck' exists to potentially eliminate severely defective mitochondria and/or mtDNA. In oocytes, the bottleneck is partly due to the manner in which mtDNA is replicated, as well as a consequence of mitochondrial organization. Oocyte mitochondria are organized into a transient structure called a Balbiani body, comprised of other organelles, such as the endoplasmic reticulum (ER) and Golgi, but the biogenesis of this structure is poorly understood $^{44}$. During the reprogramming of fibroblasts into induced pluripotent stem (iPS) cells, heteroplasmic mtDNA genotypes also segregate through a bottleneck and mitochondria are organized into a Balbiani-like structure ${ }^{45}$, suggesting that iPS cells could be a useful tool with which to study the cellular and molecular mechanisms of mtDNA genotype segregation during development.

Nuclear genes are replicated during a finite phase of the cell cycle and segregated by the concerted action of a microtubule-based spindle apparatus and an actin-based cytokinesis machine that work together to physically partition chromosomes into daughter cells. Bacterial cells also possess cell-cycle mechanisms to coordinate cell division with chromosome segregation through the placement of a tubulin-like FtsZ cell division machine. By contrast, the replication and segregation of mitochondrial chromosomes within most eukaryotes is not stringently coupled to the cell cycle, and at any given time, the replication 
of mtDNA occurs for only a subset of nucleoids in a given cell ${ }^{46}$. Bacterial cytoskeletal machinery has been lost during mitochondrial evolution, raising the question: what mechanisms are used to place division sites and segregate mtDNA? Such mechanisms will probably be important for understanding the cell- and tissue-specific mechanisms that underlie diseases linked to mtDNA mutations.

\section{Dynamin-mediated mitochondrial dynamics}

In higher eukaryotes, the segregation of mtDNA at the cellular level partly depends on continuous division and fusion events (Fig. 1c), whose rates are responsive to the needs of a particular cell type $\mathrm{e}^{47}$. One fundamental role of mitochondrial fusion is to allow communication between organelles, perhaps to facilitate access to products of mtDNA expression ${ }^{48-50}$. Mitochondrial fusion also serves as a mechanism to buffer transient defects that arise in mitochondria ${ }^{51}$. Mitochondrial division antagonizes fusion-driven network assembly to facilitate mitochondrial distribution and transport by motor proteins on cytoskeletal networks to and from distal locations of demand ${ }^{52}$. A balance between division and fusion is important for the distribution and maintenance of mtDNA. Loss of mitochondrial fusion causes a normally connected network to fragment into multiple small mitochondria owing to unopposed division, and mtDNA is either completely or partially lost from cells with the associated severe defects in oxidative phosphorylation ${ }^{50,53}$. Attenuation of mitochondrial division causes mitochondria to elongate and form highly interconnected net-like structures, as well as causing defects in oxidative phosphorylation and mtDNA loss during cell division ${ }^{54-57}$. The link between mitochondrial dynamics and mtDNA transmission is consistent with the primary role of dynamics in the control of mitochondrial copy number. The more distributive nature of mitochondrial division coupled with opposing fusion has thus evolved to replace ancestral bacterial cytoskeletal machines.

Mitochondrial division and fusion events are mediated by the action of highly conserved dynamin-related proteins (DRPs) that, through their ability to self-assemble and hydrolyse GTP, facilitate membrane remodelling of diverse intracellular membranes ${ }^{58}$. Mitochondrial division is catalysed by a single DRP, DRP1 in mammals (Dnm1 in yeast). DRP1 and Dnm1 assemble through stalk domains into helical structures that wrap around the outer surface of mitochondria at constriction sites, whose diameters match the diameters of the division helix ${ }^{59,60}$. Interactions between the GTPase domains of division DRPs across the helical rungs catalyse the hydrolysis of GTP — an event thought to produce conformational changes that are transduced through the DRP helix to allow the coordinate scission of the outer and inner membranes ${ }^{61-63}$. Fusion of the mitochondrial outer and inner membranes requires the action of two evolutionarily distinct integral membrane DRPs, MFN1/MFN2 in mammals (Fzo1 in yeast) and OPA1 in mammals (Mgm1 in yeast), respectively ${ }^{64}$. Much less is known about how fusion DRPs work at a mechanistic level, although it is likely that interactions between the GTPase domains on opposing membranes are harnessed for membrane tethering and that self-assembly through the proposed stalk-like regions within a membrane is used for fusion.

The DRP family originates in bacteria, for which evidence suggests that members also function in membrane-linked processes ${ }^{65}$. However, DRPs' acquired roles as the primary 
machines that control mitochondrial copy number are a radical divergence from the bacterial FtsZdependent division machine, which works from the cytosolic face of the plasma membrane to mediate constriction and fission (Fig. 2). Insight into this transition to DRPdriven division comes from the division machines of primitive eukaryotic organelles from organisms such as the red algae Cyanidioschyzon merolae, and from endosymbiotic plastids and chloroplasts of most photosynthetic eukaryotes. These endosymbiotic organelles possess hybrid division machines, containing both internal FtsZ and external DRP assemblies ${ }^{66,67}$. The FtsZ machine functions in constriction and scission of the inner and outer membranes and in positioning the division site, whereas the DRP machine is recruited to the outer surface of the organelle - at the constriction site - and functions relatively late in the process to complete the scission of the outer membrane. As in the case for bacteria, FtsZdependent division site placement in these mitochondria and plastids is crucial for the transmission of organellar genomes.

\section{ER-associated mitochondrial division}

The loss of the FtsZ-like machine in higher eukaryotes raises questions of how and where division sites are placed in mitochondria and whether division site placement is important for mtDNA transmission. The answers have been partially addressed by the discovery that mitochondrial division site placement is dependent on a key inter-organellar interaction with the $\mathrm{ER}^{68}$. Before DRP1 recruitment to the mitochondrial outer membrane, ER tubules wrap around mitochondria and mark sites of mitochondrial division - a phenomenon, termed ER-associated mitochondrial division (ERMD), that has been conserved from yeast to humans. At these sites, mitochondria are constricted, and thus geometric hotspots for the assembly of the division DRP helix are also created. In addition, the integral outer membrane DRP1 receptor and effector $\mathrm{MFF}^{69}$ is recruited to sites of contact, which provides a spatial mark to link DRP1 recruitment to its activation and assembly into a division machine. Neither the mechanism underlying the generation of such an ER-mitochondrial microdomain nor of ER-associated mitochondrial constriction is understood. It is possible that the ER is able to directly alter mitochondrial membrane composition and/or morphology to facilitate the recruitment of factors localized on the outside and/ or inside of mitochondria that promote mitochondrial constriction and division. In mammalian cells, the actin cytoskeleton has been implicated in ERMD, potentially through the ER-localized isoform of the formin INF2, raising the possibility that mitochondrial constriction during division is actin mediated ${ }^{70}$.

ERMD must also involve a link or tether between the two organelles. The molecular basis of this link has recently been elucidated in yeast, and is mediated by a multiprotein complex termed the ER-mitochondrial encounter structure (ERMES). This structure forms a discrete and finite number of interfaces between the ER and mitochondria in cells ${ }^{71,72}$. In addition to marking sites of division, ERMES structures are tightly linked to a subset of nucleoids engaged in replicating $\mathrm{mtDNA}^{46,73}$, potentially as components of a larger structure that spans multiple membranes. At sites of an ERMES complex, nucleoids segregate by an unknown mechanism and, in most cases, are distributed into both tips of divided mitochondria ${ }^{72}$. In this context, the ERMES complex has also been implicated as a bridge between mitochondria and the actin network, suggesting that it may link and coordinately 
drive nucleoid segregation, mitochondrial constriction during division, and mitochondrial distribution after division ${ }^{74}$. Thus, the process of ERMD and nucleoid segregation in yeast may fundamentally be related to the role of actin in ERMD in mammalian cells.

The distribution of daughter mitochondria following ERMD in yeast requires the highly conserved Miro GTPase Gem1 (ref. 72). Gem1 may function with ERMES to promote the resolution of daughter mitochondria by recruiting motility factors to mitochondrial tips after division. The metazoan Gem1 orthologues, MIRO1 and MIRO2 (hereafter referred to as Miro), also function in mitochondrial distribution. In this case, Miro proteins have been proposed to connect mitochondria to a member of the Milton/TRAK protein family of kinesin- 1 adaptors to allow the microtubule-based transport of mitochondria ${ }^{75,76}$. However, although the Miro GTPase family is remarkably conserved, the mechanisms of mitochondrial transport are divergent in eukaryotes and, in yeast, mitochondrial motility is actin dependent. Thus, it is possible that the role of Gem1 and Miro GTPases in mitochondrial distribution may instead be to allow motility by directly regulating molecular tethers to disengage mitochondria from the ER at sites of division. In any case, Gem1dependent distribution of daughter mitochondria after mitochondrial division serves as a cellular mechanism to coordinately distribute mitochondria and mtDNA.

\section{Internal determinants of mitochondrial division}

The observations discussed paint a compelling picture in which ERMD positions the division plane adjacent to mitochondrial nucleoids to bias their distribution into newly generated daughter mitochondria (Fig. 2). In mammalian cells, nucleoids are similarly localized at mitochondrial division sites and mitochondrial tips ${ }^{77,78}$, and in the absence of the division DRP DRP1, nucleoids aggregate in clusters within hyperfused mitochondria ${ }^{79}$. This suggests that ERMD's role in nucleoid distribution is conserved, although the molecular identity of the ER-mitochondrial tether or tethers at division sites in mammalian cells is unknown. More importantly, the question of what determines the placement of division sites still needs to be answered. Specifically, it is not known what determinants are required for the finite number and spatial localization of ER-mitochondria contact sites linked to nucleoid segregation. These questions are related to whether, in a manner analogous to bacterial FtsZ, there is a machine inside mitochondria that facilitates mitochondrial division. In yeast, an excellent candidate for the internal membrane scission machine is the inner membrane protein Mdm33, which possesses matrix-localized coiledcoil regions that could act in trans across inner membranes to mediate constriction ${ }^{80}$.

Given the endosymbiotic origin of mitochondria, it is tempting to speculate that a spatial mark inside the organelles is used as a divisionplane placement determinant. Evidence exists for an autonomous structure in mitochondria similar to the DNA-replicating replisome, which may function as such a mark. Specifically, nucleoid proteins required for mtDNA maintenance remain localized to discrete punctate structures within mitochondrial tubules in the absence of mtDNA in yeast and mammalian cells, suggesting that they have an intrinsic ability to organize into a structure in a mtDNA-independent manner ${ }^{46,81}$. In yeast, replisomes segregate within mitochondria and maintain their association with ERMESmarked ER-mitochondria contact sites, even in the absence of mitochondrial genomes ${ }^{46}$. 
Mitochondrial skeletal structures may also serve as internal spatial marks. Although bacterial-like cytoskeletal elements have apparently been lost, many scaffold-like structures exist within mitochondria, facilitating the formation of their complex external and internal structure. Mitochondrial scaffolds work together to create a higher-level organelle organization, which could encode spatial marks for nucleoid and/or division site placement. These scaffolds include the conserved prohibitin complex, which forms ring-like structures in the inner membrane that function together with mitochondrial lipids, such as cardiolipin and phosphatidylethanolamine, to organize inner membrane domains ${ }^{82}$. Another primary 'skeletal' element in mitochondria is the conserved multisubunit inner-membrane-associated complex MitOS (also called MICOS and MINOS) ${ }^{83-85}$. Evidence indicates that MitOS forms an extended heteromorphic structure that organizes and potentially shapes the mitochondrial inner membrane, which is differentiated into regions that are structurally, compositionally and functionally distinct. The region in close apposition to the outer membrane, termed the boundary region, facilitates lipid trafficking, mitochondrial protein import and respiratory complex assembly. Inner membrane cisternal invaginations called cristae house assembled respiratory complexes and have highly curved edges that are stabilized by the dimerization or multimerization of ATP synthase complexes ${ }^{86}$. Relatively narrow tubules, termed crista junctions, connect cristae to the boundary membrane and segregate soluble intermembrane-space components from the boundary regions. These junctions are restructured in apoptosis to promote the release of intermembranespacelocalized cell-death mediators into the cytosol during mitochondrial outer-membrane permeabilization (MOMP) ${ }^{87}$. Super-resolution imaging has revealed that mammalian nucleoids are tightly associated with inner membrane cristae ${ }^{39}$. Thus, the MitOS complex may also have a direct role in nucleoid positioning and/or may be part of a spatial mark that links the inside of mitochondria to the outside. Consistent with this possibility, in yeast, elements of the MitOS complex seem adjacent to nucleoids, and loss of an intact MitOS complex leads to nucleoid aggregation ${ }^{88}$. This complex also facilitates mitochondrial biogenesis by interacting with components of the import and sorting machineries in the outer mitochondrial membrane ${ }^{85}$. Thus, MitOS may have a more global function as a 'blueprint' of mitochondrial organization and in this capacity could act as a form-to-function integrator.

\section{The ERMD microdomain as a systems integrator}

Although the fundamental role of ER-associated division may be to control the distribution of mtDNA, evidence suggests that ERMD domains are harnessed for additional and diverse functions in cells and thus may also function as integrators. In addition to ERMD, the ERMES complex has been functionally linked to the biogenesis of outer membrane proteins and to lipid transport between the ER and mitochondria, which is crucial for the synthesis of essential mitochondrial lipids, such as phosphatidylethanolamine and cardiolipin ${ }^{71,89}$. ERMD domains thus might also monitor cellular status by facilitating communication between mitochondrial behaviours and cellular signalling pathways, for example, between mitochondrial division, fusion and cell death. Consistent with this possibility is the fact that DRP1 facilitates the recruitment and activation of the pro-apoptotic Bcl-2 protein BAX to the mitochondrial outer membrane to mediate MOMP ${ }^{90}$. Furthermore, ER-synthesized sphingolipids promote mitochondrial assembly of BAX and MOMP activation in vitro ${ }^{91}$. 
Conversely, mitochondrial fusion has a negative regulatory role in MOMP because cytosolic BAX promotes mitochondrial fusion in vitro through the DRP MFN2 (ref. 92), raising the possibility that fusion DRPs also regulate apoptosis through ERMD domains. It is possible that ERMD domains extend both into inner mitochondrial compartments and the ER lumen to integrate the functional status of both organelles as suggested by the regulation of MOMP by ER stress. Consistent with this, when ER stress occurs, the apoptotic regulator CDIP1 interacts with the ER protein BAP31, which subsequently leads to BAX assembly on mitochondria ${ }^{93}$. Thus, it will be interesting to determine whether sites of BAP31 and CDIP1 interaction coincide with sites of ERMD. The connection between the ER and mitochondria is substantiated by their roles in a shared set of diseases associated with altered mitochondrial dynamics. For example, proteins localized to ER-mitochondrial contacts have been implicated in Huntington's disease, optic atrophy and spinocerebellar ataxias ${ }^{94}$; and alteration of ER-mitochondrial contact has been described in Alzheimer's disease ${ }^{95,96}$. Thus, ERMD domain dysfunction may be a contributory factor in many diseases.

ERMD domains represent only one type of ER-mitochondrial contact. In yeast, for example, the ER is a component of two distinct Num1 and Mmr1 tethers that selectively position mitochondria at the cortex of mother and daughter cells, respectively, in a manner that is independent of ERMES and ERMD ${ }^{97,98}$. In addition, the fusion DRP MFN2, which is not essential to ERMD contact formation, has been proposed to act in mammalian cells as an ER-mitochondrial tether ${ }^{68,99}$. More work is needed, especially in mammalian cells, to understand the molecular basis of ER- mitochondria contacts and whether specialized contacts and tethers exist for different shared ER-mitochondrial functions, such as $\mathrm{Ca}^{2+}$ homeostasis, lipid biosynthesis, ERMD and for the ER-mitochondrial contacts implicated in autophagy ${ }^{100,101}$. This is an exciting area of mitochondrial biology that promises to be highly relevant to our understanding of the aetiology underlying diseases linked to mitochondrial dysfunction.

\section{Coordination of diverse mitochondrial behaviours}

Although mitochondrial division and fusion are major determinants of mitochondrial distribution, the behaviour of the mitochondrial network in cells is controlled by additional activities, such as tethering and motility. Neuronal cells are a prominent example of how these behavioural networks must work together responsively to maintain cellular function. Neurons are long, excitable cells that are highly compartmentalized and, for proper function, mitochondria must be appropriately distributed to serve the cells' different spatial and temporal demands. The demand for mitochondrial ATP production and $\mathrm{Ca}^{2+}$ buffering is especially high at axon terminals, which are dynamic structures that require the localized presence of mitochondria for synaptic transmission. Given that most mitochondrial biogenesis occurs in the soma of the neuron, active mechanisms are required to both transport and immobilize mitochondria at the distal synaptic terminals.

Insight into how these two processes are coordinated and function together to selectively target mitochondria to active synaptic terminals has come from recent studies on the neuronspecific protein syntaphilin, which binds specifically to the mitochondrial outer membrane and accumulates on immobilized axonal mitochondria localized to active terminals ${ }^{102}$. 
Mitochondria destined for axons are generated by mitochondrial division in the soma and transported to the synapse along microtubules. The spatial link between division and nucleoids, and nucleoids and cytoskeletal elements, might, in this cell type, ensure that mitochondria destined for transport contain mtDNA. Syntaphilin functions as a brake, using at least two separate mechanisms. It binds directly to the microtubule-based kinesin motor KIF5 in vitro and inhibits its motor activity, suggesting that it converts KIF5 into a component of a static microtubule-dependent mitochondrial tether. Whether the ER plays a part in the biogenesis of the syntaphilin-KIF5 tether is an outstanding question. Syntaphilin also competes for KIF5 binding with the adaptor Milton/TRAK to facilitate tethering. Thus, there is extensive interplay between the motility and tethering machines to control mitochondrial distribution in an activity dependent and spatially specific manner.

Mitochondrial dynamics are probably also coordinated on a molecular level with the transport and tethering machineries in different cell types. In mammalian cells, mitochondria that lack the fusion DRP MFN2 show severe motility defects ${ }^{103}$, similar to the motility defects observed in cells that lack Miro, and MFN2 has been reported to physically interact with Miro and Milton/TRAK ${ }^{104}$. This link between mitochondrial fusion and motility may be relevant for understanding why in humans, mutations in MFN2 and OPA1 cause the tissue-specific neurodegenerative diseases, Charcot Marie Tooth Type 2A (CMT2A) and dominant optic atrophy (DOA), respectively ${ }^{22}$. Indeed, a high frequency of mutations in mtDNA and nuclear genes that cause mitochondrial dysfunction selectively affect neurons and cause a diverse set of neurodegenerative diseases ${ }^{32}$.

\section{Mitochondrial quality control pathways}

As well as being integrated with each other, mitochondrial behaviours are integrated with a battery of stress or quality-control pathways in cells that sense and respond to mitochondrial and cellular dysfunction (Fig. 3). Inside mitochondria, molecular chaperones and quality control proteases act together to promote the assembly of protein complexes comprised of mtDNA- and nucleus-encoded proteins as well as to monitor and degrade unfolded proteins ${ }^{105}$. An imbalance between nuclear and mitochondrial proteomes and/or an accumulation of unfolded mitochondrial proteins triggers a transcriptional response program in metazoans, termed the mitochondrial unfolded protein stress response pathway (UPRmt) ${ }^{106-108}$. The response is initiated by signals produced at the mitochondrial level that activate the transcription of nucleus-encoded mitochondrial chaperone genes, as well as additional genes to restore organelle homeostasis. The pathway has been characterized at the molecular level in Caenorhabditis elegans, for which both the mitochondrial-innermembrane peptide transporter HAF1 and the bZip transcription factor ATFS1 are required for UPRmt signalling ${ }^{109}$. Recent evidence indicates that in healthy cells, ATFS1 is actively imported into the mitochondrial matrix, where it is constitutively degraded in healthy mitochondria ${ }^{110}$. Under conditions where the electron transport chain is disrupted, membrane potential is attenuated, and consequently the import efficiency of ATFS1 is decreased in a manner that is somehow dependent on HAF1. Extra-mitochondrial ATFS1 is stabilized and targeted to the nucleus, where it initiates a transcriptional response that increases the expression of mitochondrial chaperones and import machinery and remodels metabolism to rely less on respiration. Activation of the UPRmt in C. elegans is associated 
with an increase in lifespan, and recent evidence suggests that in mammals activation of this pathway also contributes to longevity, further implicating mitochondria as a crucial factor in ageing ${ }^{106}$. It remains to be determined whether the molecular mechanisms underlying the UPRmt are conserved in mammalian systems.

Additional mitochondrial stress-induced pathways are triggered by perturbations in electron transport chain function and/or reduction of membrane potential. The mitochondrial inner membrane fusion DRP OPA1 acts as a toggle between two pathways. In healthy cells, OPA1 processing occurs constitutively by the i-AAA protease YME1L to generate long transmembrane anchored and short, soluble isoforms, which are required for membrane fusion ${ }^{111}$. Reduction of mitochondrial membrane potential causes the long OPA1 isoforms to be converted into short forms by the metalloprotease OMA1, resulting in the inhibition of mitochondrial fusion and subsequent mitochondrial fragmentation ${ }^{112,113}$. This alteration serves to potentiate mitophagy and/or cell death. Conversely, long OPA1 isoforms are required for a different stress-induced response, termed mitochondrial hyperfusion. Mitochondrial hyperfusion mediates the formation of a highly connected mitochondrial network and is thus thought to buffer the potentially deleterious effects of stresses, including those caused by ultraviolet irradiation and nutrient starvation. In the case of starvation, hyperfusion has been proposed to protect mitochondria from autophagic degradation or mitophagy through steric hinderance ${ }^{114,115}$. More recent work indicates that mitochondrial hyperfusion also serves as a homeostatic response to maintain ATP production in cases where complex IV of the electron transport chain is impaired ${ }^{51}$. The hyperfusion response, however, is transient and thus cannot buffer long-term defects in electron transport chain activity. A more terminal response to mitochondrial dysfunction is mitophagy, which is also triggered by a decrease in membrane-potential-driven protein import. In this pathway, the kinase PINK1 is imported into healthy mitochondria and constitutively degraded. A decrease in import triggered by mitochondrial dysfunction causes PINK1 to accumulate on the outer membrane, where it recruits the E3 ligase Parkin 116,117. Among Parkin's targets for ubiquitination are the transport factor Miro and the mitochondrial fusion protein MFN2 (ref. 118-120). The proteasomal degradation of ubiquitinated mitochondrial outer membrane proteins is dependent on the AAA-ATPase p97 in a manner analogous to ER-associated degradation $^{120,121}$. The Parkin-dependent degradation of factors involved in mitochondrial motility and fusion enhances the selectivity of removing defective mitochondria by autophagy. In addition to specifying defective mitochondria for degradation, an in vivo study in Drosophila suggests that the PINK1-Parkin pathway may also be capable of selectively targeting respiratory complexes for degradation ${ }^{122}$. In support of this idea, the selective targeting of complex I for degradation has also been described in cell culture models ${ }^{45}$, but the mechanisms underlying this phenomenon are currently unknown.

Mitochondrial stress pathways probably have important roles in disease manifestations of mitochondrial dysfunction and, as such, could highlight promising therapeutic targets. In the case of the PINK1-Parkin mitophagy pathway, mutations in each gene are linked to genetically inherited Parkinson's disease ${ }^{123,124}$, implying the pathway is relevant. However, most of the stress pathways described above, particularly those caused by a loss of membrane potential, have been characterized under conditions of acute extreme stress, 
raising the question of their physiological relevance ${ }^{125}$. In addition, despite these numerous stress pathways, mtDNA mutations are able to accumulate, particularly in differentiated post-mitotic cells, at the expense of functional wild-type mtDNA, leading to disease states. Thus, future work should focus on developing animal models that mimic diseases associated with mitochondrial dysfunction to assess the physiological contributions of these pathways. In addition, continued basic biological approaches are necessary to assess how the cell appropriately senses, and differentially activates and coordinates these pathways with each other and with other signalling pathways, such as those involved in cell death (Fig. 3). For example, it is unclear how cells appropriately integrate the UPRmt and mitophagy pathways, which are both regulated at the basic level of import. It is also unknown whether OPA1 can function as a molecular integrator of stresses or simply as a modulator of mitochondrial shape or how OPA1-dependent stress pathways are coordinated with UPRmt and mitophagy.

The emerging picture of mitochondria is that of 'super-organized' structural domains for building an organelle whose behaviour is wired to be responsive to cellular needs, as well as its own dysfunction. The combined use of system-based approaches, with super-resolution microscopy and new genetic tools will allow us to understand the molecular basis of mitochondrial structure in detail. Exactly how mitochondrial super-organization is constructed will address the fundamental question of whether primary determinants of organization originate from the inside of the organelle and are intimately tied to the most ancient feature - the genome.

\section{Acknowledgments}

We thank members of the Nunnari lab for helpful discussions and comments. We also thank K. Osteryoung and S. Lewis for helpful discussions. J.N. is supported by NIH grants R01GM062942, R01GM097432 and R01GM106019. J.F. is supported by a fellowship from the Jane Coffin Childs Memorial Fund for Medical Research.

\section{References}

1. Lane N, Martin W. The energetics of genome complexity. Nature. 2010; 467:929-934. [PubMed: 20962839]

2. Gabaldón T, Huynen MA. Shaping the mitochondrial proteome. Biochim. Biophys. Acta. 2004; 1659:212-220. [PubMed: 15576054]

3. Baughman JM, et al. Integrative genomics identifies MCU as an essential component of the mitochondrial calcium uniporter. Nature. 2011; 476:341-345. [PubMed: 21685886]

4. De Stefani D, Raffaello A, Teardo E, Szabo I, Rizzuto R. A forty-kilodalton protein of the inner membrane is the mitochondrial calcium uniporter. Nature. 2011; 476:336-340. [PubMed: 21685888]

5. Pagliarini DJ, et al. A mitochondrial protein compendium elucidates complex I disease biology. Cell. 2008; 134:112-123. [PubMed: 18614015]

6. Sickmann A, et al. The proteome of Saccharomyces cerevisiae mitochondria. Proc. Natl Acad. Sci. USA. 2003; 100:13207-13212. [PubMed: 14576278]

7. Forner F, Foster LJ, Campanaro S, Valle G, Mann M. Quantitative proteomic comparison of rat mitochondria from muscle, heart, and liver. Mol. Cell. Proteomics. 2006; 5:608-619. [PubMed: 16415296]

8. Lecrenier N, Van Der Bruggen P, Foury F. Mitochondrial DNA polymerases from yeast to man: a new family of polymerases. Gene. 1997; 185:147-152. [PubMed: 9034326] 
9. Stumpf JD, Copeland WC. Mitochondrial DNA replication and disease: insights from DNA polymerase $\gamma$ mutations. Cellular and molecular life sciences. Cell. Mol. Life Sci. 2011; 68:219233. [PubMed: 20927567]

10. Tiranti V, et al. Identification of the gene encoding the human mitochondrial RNA polymerase (hmtRPOL) by cyberscreening of the expressed sequence tags database. Hum. Mol. Genet. 1997; 6:615-625. [PubMed: 9097968]

11. Christian BE, Spremulli LL. Mechanism of protein biosynthesis in mammalian mitochondria. Biochim. Biophys. Acta. 2012; 1819:1035-1054. [PubMed: 22172991]

12. Fung S, Nishimura T, Sasarman F, Shoubridge EA. The conserved interaction of C7orf30 with MRPL14 promotes biogenesis of the mitochondrial large ribosomal subunit and mitochondrial translation. Mol. Biol. Cell. 2013; 24:184-193. [PubMed: 23171548]

13. Sharma MR, et al. Structure of the mammalian mitochondrial ribosome reveals an expanded functional role for its component proteins. Cell. 2003; 115:97-108. [PubMed: 14532006]

14. Neupert W, Herrmann JM. Translocation of proteins into mitochondria. Annu. Rev. Biochem. 2007; 76:723-749. [PubMed: 17263664]

15. Schmidt O, Pfanner N, Meisinger C. Mitochondrial protein import: from proteomics to functional mechanisms. Nature Rev. Mol. Cell Biol. 2010; 11:655-667. [PubMed: 20729931]

16. Jäger S, Handschin C, St-Pierre J, Spiegelman BM. AMP-activated protein kinase (AMPK) action in skeletal muscle via direct phosphorylation of PGC-1a. Proc. Natl Acad. Sci. USA. 2007; 104:12017-12022. [PubMed: 17609368]

17. Jeninga EH, Schoonjans K, Auwerx J. Reversible acetylation of PGC-1: connecting energy sensors and effectors to guarantee metabolic flexibility. Oncogene. 2010; 29:4617-4624. [PubMed: 20531298]

18. Scarpulla RC, Vega RB, Kelly DP. Transcriptional integration of mitochondrial biogenesis. Trends Endocrinol. Metab. 2012; 23:459-466. [PubMed: 22817841]

19. Gadir N, Haim-Vilmovsky L, Kraut-Cohen J, Gerst JE. Localization of mRNAs coding for mitochondrial proteins in the yeast Saccharomyces cerevisiae. RNA. 2011; 17:1551-1565. [PubMed: 21705432]

20. Garcia M, et al. Mitochondria-associated yeast mRNAs and the biogenesis of molecular complexes. Mol. Biol. Cell. 2007; 18:362-368. [PubMed: 17108321]

21. Schmidt O, et al. Regulation of mitochondrial protein import by cytosolic kinases. Cell. 2011; 144:227-239. [PubMed: 21215441]

22. Nunnari J, Suomalainen A. Mitochondria: in sickness and in health. Cell. 2012; 148:1145-1159. [PubMed: 22424226]

23. Wallace DC, et al. Mitochondrial DNA mutation associated with Leber's hereditary optic neuropathy. Science. 1988; 242:1427-1430. [PubMed: 3201231]

24. Morris AA, et al. Deficiency of respiratory chain complex I is a common cause of Leigh disease. Ann. Neurol. 1996; 40:25-30. [PubMed: 8687187]

25. Ross JM, et al. Germline mitochondrial DNA mutations aggravate ageing and can impair brain development. Nature. 2013; 501:412-415. [PubMed: 23965628]

26. Ahlqvist KJ, et al. Somatic progenitor cell vulnerability to mitochondrial DNA mutagenesis underlies progeroid phenotypes in Polg mutator mice. Cell Metab. 2012; 15:100-109. [PubMed: 22225879]

27. Ngo HB, Kaiser JT, Chan DC. The mitochondrial transcription and packaging factor Tfam imposes a U-turn on mitochondrial DNA. Nature Struct. Mol. Biol. 2011; 18:1290-1296. [PubMed: 22037171]

28. Rubio-Cosials A, et al. Human mitochondrial transcription factor A induces a U-turn structure in the light strand promoter. Nature Struct. Mol. Biol. 2011; 18:1281-1289. [PubMed: 22037172]

29. Ekstrand MI, et al. Mitochondrial transcription factor A regulates mtDNA copy number in mammals. Hum. Mol. Genet. 2004; 13:935-944. [PubMed: 15016765]

30. Shi Y, et al. Mammalian transcription factor A is a core component of the mitochondrial transcription machinery. Proc. Natl Acad. Sci. USA. 2012; 109:16510-16515. [PubMed: 23012404] 
31. Bogenhagen DF. Mitochondrial DNA nucleoid structure. Biochim. Biophys. Acta. 2012; 1819:914-920. [PubMed: 22142616]

32. Copeland WC. Defects in mitochondrial DNA replication and human disease. Crit. Rev. Biochem. Mol. Biol. 2012; 47:64-74. [PubMed: 22176657]

33. Bogenhagen DF, Wang Y, Shen EL, Kobayashi R. Protein components of mitochondrial DNA nucleoids in higher eukaryotes. Mol. Cell. Proteomics. 2003; 2:1205-1216. [PubMed: 14514796]

34. Kaufman BA, et al. In organello formaldehyde crosslinking of proteins to mtDNA: identification of bifunctional proteins. Proc. Natl Acad. Sci. USA. 2000; 97:7772-7777. [PubMed: 10869431]

35. He J, et al. Mitochondrial nucleoid interacting proteins support mitochondrial protein synthesis. Nucleic Acids Res. 2012; 40:6109-6121. [PubMed: 22453275]

36. Mbantenkhu M, et al. Mgm101 is a Rad52-related protein required for mitochondrial DNA recombination. J. Biol. Chem. 2011; 286:42360-42370. [PubMed: 22027892]

37. Holt IJ, Reyes A. Human mitochondrial DNA replication. Cold Spring Harb. Perspect. Biol. 2012; 4:a012971. [PubMed: 23143808]

38. Brown TA, Tkachuk AN, Clayton DA. Native R-loops persist throughout the mouse mitochondrial DNA genome. J. Biol. Chem. 2008; 283:36743-36751. [PubMed: 18986989]

39. Brown TA, et al. Superresolution fluorescence imaging of mitochondrial nucleoids reveals their spatial range, limits, and membrane interaction. Mol. Cell. Biol. 2011; 31:4994-5010. [PubMed: 22006021]

40. Kukat C, et al. Super-resolution microscopy reveals that mammalian mitochondrial nucleoids have a uniform size and frequently contain a single copy of mtDNA. Proc. Natl Acad. Sci. USA. 2011; 108:13534-13539. [PubMed: 21808029]

41. Birky CW Jr. The inheritance of genes in mitochondria and chloroplasts: laws, mechanisms, and models. Annu. Rev. Genet. 2001; 35:125-148. [PubMed: 11700280]

42. Al Rawi S, et al. Postfertilization autophagy of sperm organelles prevents paternal mitochondrial DNA transmission. Science. 2011; 334:1144-1147. [PubMed: 22033522]

43. Sato M, Sato K. Degradation of paternal mitochondria by fertilizationtriggered autophagy in $C$. elegans embryos. Science. 2011; 334:1141-1144. [PubMed: 21998252]

44. Pepling ME, Wilhelm JE, O’Hara AL, Gephardt GW, Spradling AC. Mouse oocytes within germ cell cysts and primordial follicles contain a Balbiani body. Proc. Natl Acad. Sci. USA. 2007; 104:187-192. [PubMed: 17189423]

45. Hämäläinen RH, et al. Tissue- and cell-type-specific manifestations of heteroplasmic mtDNA $3243 \mathrm{~A}>\mathrm{G}$ mutation in human induced pluripotent stem cell-derived disease model. Proc. Natl Acad. Sci. USA. 2013; 110:E3622-E3630. [PubMed: 24003133] This paper showed that during reprogramming of heteroplasmic fibroblasts derived from mitochondrial disease patients, mutant and wild-type mitochondrial genomes segregate through a bottleneck towards a homoplasmic state.

46. Meeusen S, Nunnari J. Evidence for a two membrane-spanning autonomous mitochondrial DNA replisome. J. Cell Biol. 2003; 163:503-510. [PubMed: 14597773]

47. Hoppins S, Lackner L, Nunnari J. The machines that divide and fuse mitochondria. Annu. Rev. Biochem. 2007; 76:751-780. [PubMed: 17362197]

48. Chen H, McCaffery JM, Chan DC. Mitochondrial fusion protects against neurodegeneration in the cerebellum. Cell. 2007; 130:548-562. [PubMed: 17693261]

49. Chen $\mathrm{H}$, et al. Mitochondrial fusion is required for mtDNA stability in skeletal muscle and tolerance of mtDNA mutations. Cell. 2010; 141:280-289. [PubMed: 20403324]

50. Hermann GJ, et al. Mitochondrial fusion in yeast requires the transmembrane GTPase Fzo1p. J. Cell Biol. 1998; 143:359-373. [PubMed: 9786948]

51. Rolland SG, et al. Impaired complex IV activity in response to loss of LRPPRC function can be compensated by mitochondrial hyperfusion. Proc. Natl Acad. Sci. USA. 2013; 110:E2967-E2976. [PubMed: 23878239] This paper demonstrates that mitochondria can undergo hyperfusion and temporarily maintain ATP production to compensate for a reduction of complex IV activity due to loss of the RNA-binding protein LRPPRC.

52. Verstreken P, et al. Synaptic mitochondria are critical for mobilization of reserve pool vesicles at Drosophila neuromuscular junctions. Neuron. 2005; 47:365-378. [PubMed: 16055061] 
53. Chen H, Chomyn A, Chan DC. Disruption of fusion results in mitochondrial heterogeneity and dysfunction. J. Biol. Chem. 2005; 280:26185-26192. [PubMed: 15899901]

54. Ishihara N, et al. Mitochondrial fission factor Drp1 is essential for embryonic development and synapse formation in mice. Nature Cell Biol. 2009; 11:958-966. [PubMed: 19578372]

55. Parone PA, et al. Preventing mitochondrial fission impairs mitochondrial function and leads to loss of mitochondrial DNA. PLoS ONE. 2008; 3:e3257. [PubMed: 18806874]

56. Wakabayashi J, et al. The dynamin-related GTPase Drp1 is required for embryonic and brain development in mice. J. Cell Biol. 2009; 186:805-816. [PubMed: 19752021]

57. Hanekamp T, et al. Maintenance of mitochondrial morphology is linked to maintenance of the mitochondrial genome in Saccharomyces cerevisiae. Genetics. 2002; 162:1147-1156. [PubMed: 12454062]

58. Faelber K, et al. Oligomerization of dynamin superfamily proteins in health and disease. Prog. Mol. Biol. Transl. Sci. 2013; 117:411-443. [PubMed: 23663977]

59. Ingerman E, et al. Dnm1 forms spirals that are structurally tailored to fit mitochondria. J. Cell Biol. 2005; 170:1021-1027. [PubMed: 16186251]

60. Labrousse AM, Zappaterra MD, Rube DA, van der Bliek AM. C. elegans dynamin-related protein DRP-1 controls severing of the mitochondrial outer membrane. Mol. Cell. 1999; 4:815-826. [PubMed: 10619028]

61. Ford MG, Jenni S, Nunnari J. The crystal structure of dynamin. Nature. 2011; 477:561-566. [PubMed: 21927001]

62. Fröhlich C, et al. Structural insights into oligomerization and mitochondrial remodelling of dynamin 1-like protein. EMBO J. 2013; 32:1280-1292. [PubMed: 23584531]

63. Faelber K, et al. Crystal structure of nucleotide-free dynamin. Nature. 2011; 477:556-560. [PubMed: 21927000]

64. Meeusen S, McCaffery JM, Nunnari J. Mitochondrial fusion intermediates revealed in vitro. Science. 2004; 305:1747-1752. [PubMed: 15297626]

65. Low HH, Sachse C, Amos LA, Lowe J. Structure of a bacterial dynaminlike protein lipid tube provides a mechanism for assembly and membrane curving. Cell. 2009; 139:1342-1352. [PubMed: 20064379]

66. Osteryoung KW, Nunnari J. The division of endosymbiotic organelles. Science. 2003; 302:16981704. [PubMed: 14657485]

67. Nishida K, et al. Dynamic recruitment of dynamin for final mitochondrial severance in a primitive red alga. Proc. Natl Acad. Sci. USA. 2003; 100:2146-2151. [PubMed: 12566569]

68. Friedman JR, et al. ER tubules mark sites of mitochondrial division. Science. 2011; 334:358-362. [PubMed: 21885730]

69. Otera $\mathrm{H}$, et al. Mff is an essential factor for mitochondrial recruitment of Drp1 during mitochondrial fission in mammalian cells. J. Cell Biol. 2010; 191:1141-1158. [PubMed: 21149567]

70. Korobova F, Ramabhadran V, Higgs HN. An actin-dependent step in mitochondrial fission mediated by the ER-associated formin INF2. Science. 2013; 339:464-467. [PubMed: 23349293]

71. Kornmann B, et al. An ER-mitochondria tethering complex revealed by a synthetic biology screen. Science. 2009; 325:477-481. [PubMed: 19556461]

72. Murley A, et al. ER-associated mitochondrial division links the distribution of mitochondria and mitochondrial DNA in yeast. eLife. 2013; 2:e00422. [PubMed: 23682313] In yeast, ERMD serves to segregate mitochondrial genomes into tips of newly divided mitochondria, and the conserved Miro GTPase Gem1 may spatially resolve ER-mitochondrial contacts post-division.

73. Hobbs AE, Srinivasan M, McCaffery JM, Jensen RE. Mmm1p, a mitochondrial outer membrane protein, is connected to mitochondrial DNA (mtDNA) nucleoids and required for mtDNA stability. J. Cell Biol. 2001; 152:401-410. [PubMed: 11266455]

74. Boldogh IR, et al. A protein complex containing Mdm10p, Mdm12p, and Mmm1p links mitochondrial membranes and DNA to the cytoskeleton-based segregation machinery. Mol. Biol. Cell. 2003; 14:4618-4627. [PubMed: 13679517] 
75. Glater EE, Megeath LJ, Stowers RS, Schwarz TL. Axonal transport of mitochondria requires milton to recruit kinesin heavy chain and is light chain independent. J. Cell Biol. 2006; 173:545557. [PubMed: 16717129]

76. Fransson S, Ruusala A, Aspenstrom P. The atypical Rho GTPases Miro-1 and Miro-2 have essential roles in mitochondrial trafficking. Biochem. Biophys. Res. Commun. 2006; 344:500 510. [PubMed: 16630562]

77. Iborra FJ, Kimura H, Cook PR. The functional organization of mitochondrial genomes in human cells. BMC Biol. 2004; 2:9. [PubMed: 15157274]

78. Garrido N, et al. Composition and dynamics of human mitochondrial nucleoids. Mol. Biol. Cell. 2003; 14:1583-1596. [PubMed: 12686611]

79. Ban-Ishihara R, Ishihara T, Sasaki N, Mihara K, Ishihara N. Dynamics of nucleoid structure regulated by mitochondrial fission contributes to cristae reformation and release of cytochrome $c$. Proc. Natl Acad. Sci. USA. 2013; 110:11863-11868. [PubMed: 23821750]

80. Messerschmitt M, et al. The inner membrane protein Mdm33 controls mitochondrial morphology in yeast. J. Cell Biol. 2003; 160:553-564. [PubMed: 12591915]

81. Spelbrink JN, et al. Human mitochondrial DNA deletions associated with mutations in the gene encoding Twinkle, a phage T7 gene 4-like protein localized in mitochondria. Nature Genet. 2001; 28:223-231. [PubMed: 11431692]

82. Osman C, Voelker DR, Langer T. Making heads or tails of phospholipids in mitochondria. J. Cell Biol. 2011; 192:7-16. [PubMed: 21220505]

83. Hoppins $\mathrm{S}$, et al. A mitochondrial-focused genetic interaction map reveals a scaffold-like complex required for inner membrane organization in mitochondria. J. Cell Biol. 2011; 195:323-340. [PubMed: 21987634]

84. Harner M, et al. The mitochondrial contact site complex, a determinant of mitochondrial architecture. EMBO J. 2011; 30:4356-4370. [PubMed: 22009199]

85. von der Malsburg K, et al. Dual role of mitofilin in mitochondrial membrane organization and protein biogenesis. Dev. Cell. 2011; 21:694-707. [PubMed: 21944719]

86. Davies KM, Anselmi C, Wittig I, Faraldo-Gomez JD, Kuhlbrandt W. Structure of the yeast F1FoATP synthase dimer and its role in shaping the mitochondrial cristae. Proc. Natl Acad. Sci. USA. 2012; 109:13602-13607. [PubMed: 22864911]

87. Frezza C, et al. OPA1 controls apoptotic cristae remodeling independently from mitochondrial fusion. Cell. 2006; 126:177-189. [PubMed: 16839885]

88. Itoh K, Tamura Y, Iijima M, Sesaki H. Effects of Fcj1-Mos1 and mitochondrial division on aggregation of mitochondrial DNA nucleoids and organelle morphology. Mol. Biol. Cell. 2013; 24:1842-1851. [PubMed: 23615445]

89. Voss C, Lahiri S, Young BP, Loewen CJ, Prinz WA. ER-shaping proteins facilitate lipid exchange between the ER and mitochondria in S. cerevisiae. J. Cell Sci. 2012; 125:4791-4799. [PubMed: 22797914]

90. Montessuit S, et al. Membrane remodeling induced by the dynamin-related protein Drp1 stimulates Bax oligomerization. Cell. 2010; 142:889-901. [PubMed: 20850011]

91. Chipuk JE, et al. Sphingolipid metabolism cooperates with BAK and BAX to promote the mitochondrial pathway of apoptosis. Cell. 2012; 148:988-1000. [PubMed: 22385963]

92. Hoppins S, et al. The soluble form of Bax regulates mitochondrial fusion via MFN2 homotypic complexes. Mol. Cell. 2011; 41:150-160. [PubMed: 21255726]

93. Namba T, et al. CDIP1-BAP31 complex transduces apoptotic signals from endoplasmic reticulum to mitochondria under endoplasmic reticulum stress. Cell Rep. 2013; 5:331-339. [PubMed: 24139803]

94. Schon EA, Przedborski S. Mitochondria: the next (neurode) generation. Neuron. 2011; 70:10331053. [PubMed: 21689593]

95. Area-Gomez E, et al. Upregulated function of mitochondria-associated ER membranes in Alzheimer disease. EMBO J. 2012; 31:4106-4123. [PubMed: 22892566]

96. Hedskog L, et al. Modulation of the endoplasmic reticulum-mitochondria interface in Alzheimer's disease and related models. Proc. Natl Acad. Sci. USA. 2013; 110:7916-7921. [PubMed: 23620518] 
97. Lackner LL, Ping H, Graef M, Murley A, Nunnari J. Endoplasmic reticulum-associated mitochondria-cortex tether functions in the distribution and inheritance of mitochondria. Proc. Natl Acad. Sci. USA. 2013; 110:E458-E467. [PubMed: 23341591]

98. Swayne TC, et al. Role for cER and Mmrlp in anchorage of mitochondria at sites of polarized surface growth in budding yeast. Curr. Biol. 2011; 21:1994-1999. [PubMed: 22119524]

99. de Brito OM, Scorrano L. Mitofusin 2 tethers endoplasmic reticulum to mitochondria. Nature. 2008; 456:605-610. [PubMed: 19052620]

100. Rowland AA, Voeltz GK. Endoplasmic reticulum-mitochondria contacts: function of the junction. Nature Rev. Mol. Cell Biol. 2012; 13:607-625. [PubMed: 22992592]

101. Hamasaki M, et al. Autophagosomes form at ER-mitochondria contact sites. Nature. 2013; 495:389-393. [PubMed: 23455425]

102. Chen Y, Sheng ZH. Kinesin-1-syntaphilin coupling mediates activity-dependent regulation of axonal mitochondrial transport. J. Cell Biol. 2013; 202:351-364. [PubMed: 23857772] The authors show that the protein syntaphilin can regulate mitochondrial position in neurons by acting as a molecular brake through its binding to the microtubule motor Kif5

103. Baloh RH, Schmidt RE, Pestronk A, Milbrandt J. Altered axonal mitochondrial transport in the pathogenesis of Charcot-Marie-Tooth disease from mitofusin 2 mutations. J. Neurosci. 2007; 27:422-430. [PubMed: 17215403]

104. Misko A, Jiang S, Wegorzewska I, Milbrandt J, Baloh RH. Mitofusin 2 is necessary for transport of axonal mitochondria and interacts with the Miro/Milton complex. J. Neurosci. 2010; 30:42324240. [PubMed: 20335458]

105. Baker MJ, Tatsuta T, Langer T. Quality control of mitochondrial proteostasis. Cold Spring Harb. Perspect. Biol. 2011; 3:a007559. [PubMed: 21628427]

106. Houtkooper RH, et al. Mitonuclear protein imbalance as a conserved longevity mechanism. Nature. 2013; 497:451-457. [PubMed: 23698443]

107. Zhao Q, et al. A mitochondrial specific stress response in mammalian cells. EMBO J. 2002; 21:4411-4419. [PubMed: 12198143]

108. Martinus RD, et al. Selective induction of mitochondrial chaperones in response to loss of the mitochondrial genome. Eur. J. Biochem. 1996; 240:98-103. [PubMed: 8797841]

109. Haynes CM, Yang Y, Blais SP, Neubert TA, Ron D. The matrix peptide exporter HAF-1 signals a mitochondrial UPR by activating the transcription factor ZC376.7 in C. elegans. Mol. Cell. 2010; 37:529-540. [PubMed: 20188671]

110. Nargund AM, Pellegrino MW, Fiorese CJ, Baker BM, Haynes CM. Mitochondrial import efficiency of ATFS-1 regulates mitochondrial UPR activation. Science. 2012; 337:587-590.

[PubMed: 22700657] This work demonstrated that in C. elegans, the transcription factor ATFS-1 senses and modulates a response to mitochondrial stress through its targeting to either the mitochondrial matrix or the nucleus.

111. Griparic L, Kanazawa T, van der Bliek AM. Regulation of the mitochondrial dynamin-like protein Opa1 by proteolytic cleavage. J. Cell Biol. 2007; 178:757-764. [PubMed: 17709430]

112. Head B, Griparic L, Amiri M, Gandre-Babbe S, van der Bliek AM. Inducible proteolytic inactivation of OPA1 mediated by the OMA1 protease in mammalian cells. J. Cell Biol. 2009; 187:959-966. [PubMed: 20038677]

113. Ehses $\mathrm{S}$, et al. Regulation of OPA1 processing and mitochondrial fusion by m-AAA protease isoenzymes and OMA1. J. Cell Biol. 2009; 187:1023-1036. [PubMed: 20038678]

114. Rambold AS, Kostelecky B, Elia N, Lippincott-Schwartz J. Tubular network formation protects mitochondria from autophagosomal degradation during nutrient starvation. Proc. Natl Acad. Sci. USA. 2011; 108:10190-10195. [PubMed: 21646527]

115. Gomes LC, Di Benedetto G, Scorrano L. During autophagy mitochondria elongate, are spared from degradation and sustain cell viability. Nature Cell Biol. 2011; 13:589-598. [PubMed: 21478857]

116. Matsuda N, et al. PINK1 stabilized by mitochondrial depolarization recruits Parkin to damaged mitochondria and activates latent Parkin for mitophagy. J. Cell Biol. 2010; 189:211-221. [PubMed: 20404107] 
117. Narendra DP, et al. PINK1 is selectively stabilized on impaired mitochondria to activate Parkin. PLoS Biol. 2010; 8:e1000298. [PubMed: 20126261]

118. Wang X, et al. PINK1 and Parkin target Miro for phosphorylation and degradation to arrest mitochondrial motility. Cell. 2011; 147:893-906. [PubMed: 22078885]

119. Chan NC, et al. Broad activation of the ubiquitin-proteasome system by Parkin is critical for mitophagy. Hum. Mol. Genet. 2011; 20:1726-1737. [PubMed: 21296869]

120. Tanaka A, et al. Proteasome and p97 mediate mitophagy and degradation of mitofusins induced by Parkin. J. Cell Biol. 2010; 191:1367-1380. [PubMed: 21173115]

121. Xu S, Peng G, Wang Y, Fang S, Karbowski M. The AAA-ATPase p97 is essential for outer mitochondrial membrane protein turnover. Mol. Biol. Cell. 2011; 22:291-300. [PubMed: 21118995]

122. Vincow ES, et al. The PINK1-Parkin pathway promotes both mitophagy and selective respiratory chain turnover in vivo. Proc. Natl Acad. Sci. USA. 2013; 110:6400-6405. [PubMed: 23509287] The authors utilized proteomics of Drosophila Pink1 and Parkin mutants to show that respiratory complex components are selectively turned over compared with other mitochondrial proteins during mitophagy.

123. Kitada T, et al. Mutations in the parkin gene cause autosomal recessive juvenile parkinsonism. Nature. 1998; 392:605-608. [PubMed: 9560156]

124. Valente EM, et al. Hereditary early-onset Parkinson's disease caused by mutations in PINK1. Science. 2004; 304:1158-1160. [PubMed: 15087508]

125. Sterky FH, Lee S, Wibom R, Olson L, Larsson NG. Impaired mitochondrial transport and Parkinindependent degeneration of respiratory chain-deficient dopamine neurons in vivo. Proc. Natl Acad. Sci. USA. 2011; 108:12937-12942. [PubMed: 21768369] 

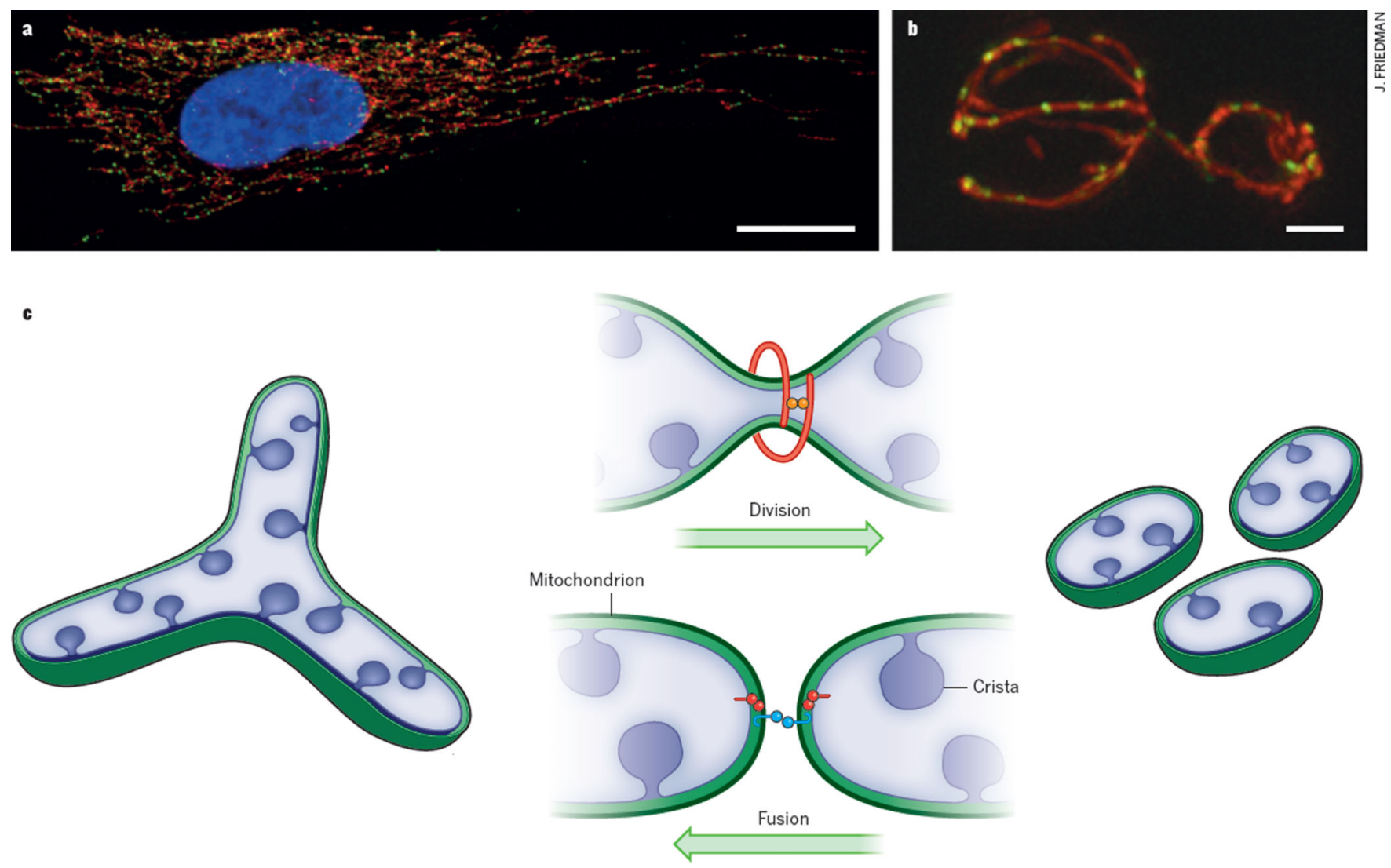

Figure 1. The organization and distribution of mitochondria and mtDNA in higher eukaryotes Mitochondrial organization is a conserved feature. a, mtDNA in a human fibroblast is packaged within nucleoids (green) distributed within tubular mitochondria (red) around the nucleus (blue). Scale bar, 20 microns. Adapted with permission from ref. 40. b, A similar distribution is seen in a yeast cell with nucleoids (green) within mitochondria (red). Scale bar, 2 microns. c, Mitochondrial copy number is controlled by the combined actions of mitochondrial division and fusion. Mitochondrial division is controlled by the assembly of a dynamin-related protein (DRP) on the outside of the organelle into a helical structure, which mediates scission through interactions across helical rungs (marked by orange circles).

Mitochondrial fusion is controlled by interactions of outer and inner membrane fusion DRPs (blue and red circles, respectively) across mitochondrial membranes. 
a

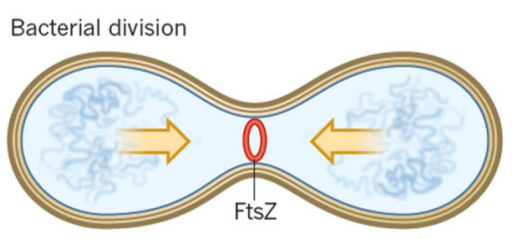

Chloroplast, plastid or primitive mitochondrion

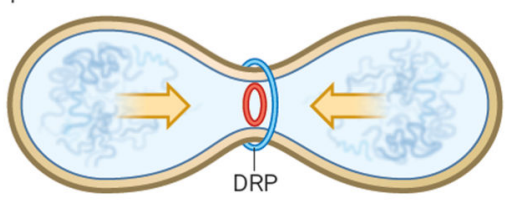

Mitochondrion

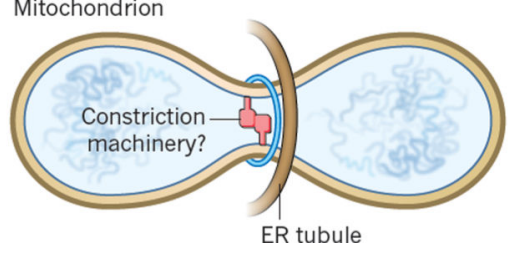

b

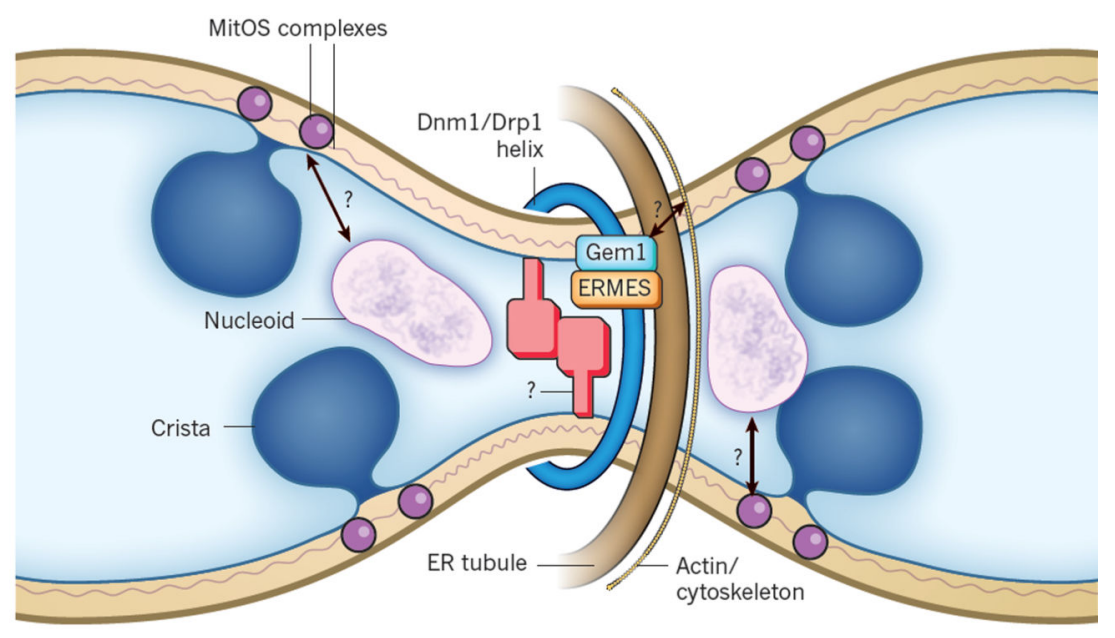

Figure 2. Evolution of mitochondrial division site placement mechanisms

a, Roles of FtsZ and dynamin-related proteins (DRPs) in bacterial and endosymbiotic organelle division and division site placement. In the a-proteobacterial ancestor of mitochondria, an FtsZ ring is placed mid-cell by active mechanisms. The combined actions of the FtsZ-containing ring and cell-wall synthesis are essential for cell division. In chloroplasts and algal mitochondria, FtsZ-dependent placement (indicated by arrows) and division mechanisms on the inside of the organelle have been retained during evolution. However, in these organelles, DRPs also function on the cytosolic surface in organelle division, perhaps replacing the requirement for cell-wall synthesis in division. In yeast or animals, DRPs function on the cytosolic surface in organelle division. Before DRP recruitment, however, the endoplasmic reticulum (ER) is associated with division-site placement and constriction on the outside of the organelle, potentially replacing FtsZdependent placement and constriction. b, Molecular model for division site placement coupled to nucleoid segregation in yeast mitochondria. In yeast, the ER-mitochondria tethering complex, ER-mitochondrial encounter structure (ERMES), and the conserved Miro GTPase Gem1 are spatially and functionally linked to ER-associated mitochondrial division (ERMD). ERMD sites marked by these components are also spatially localized to a subset of nucleoids that are actively replicating, and these segregate before ERMD. Gem1 acts as a negative regulator of ERMES-dependent ER-mitochondria contacts and is required for the spatial resolution of newly generated mitochondria by ERMD, possibly through the localized recruitment of the actin cytoskeleton. Cytoskeletal components may also participate in ER-associated mitochondrial constriction before DRP recruitment. Nucleoid placement at sites of ERMD could be mediated by a mark inside the organelle formed by the scaffold MitOS complex. Mdm33 is a possible candidate for the internal membrane scission machine in yeast. 


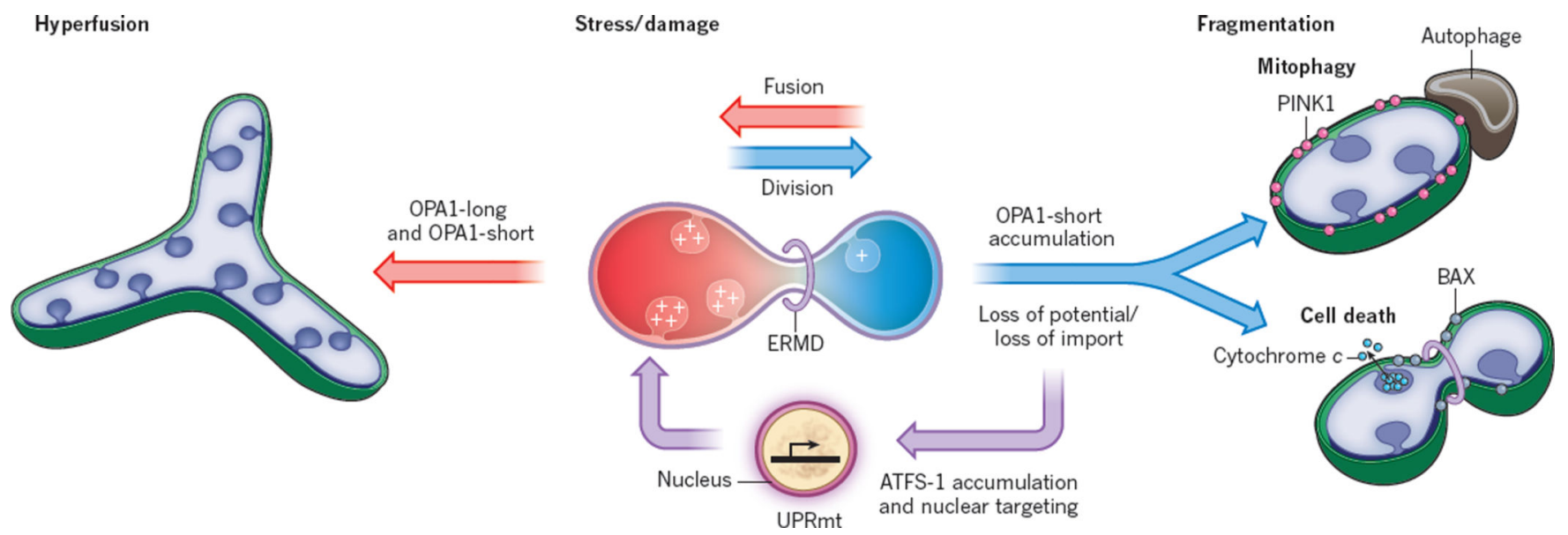

Figure 3. Integration of mitochondrial stress response pathways and their coordination with mitochondrial shape

Several different mitochondrial pathways respond to stress or damage and are coordinated with mitochondrial dynamics. Mitochondria in healthy cells generate an electrochemical potential that serves in oxidative phosphorylation and drives the import of proteins into the organelle. Damage that leads to a loss (blue) of mitochondrial membrane potential can lead to a loss of protein import efficiency. In the unfolded protein stress response (UPRmt) pathway, loss of import leads to the accumulation of the transcription factor ATFS1 in the nucleus, activating a transcriptional mitochondrial repair and metabolic adaptation response. Loss of membrane potential also triggers the OMA1-dependent proteolysis of long isoforms of the inner membrane fusion DRP OPA1, which attenuates mitochondrial fusion and potentially increases ER-mediated mitochondrial division (ERMD), resulting in mitochondrial fragmentation. These fragmented mitochondria that have lost the ability to respire and import may also accumulate the PINK1 kinase, which triggers mitophagy. In addition, under these conditions, the ERMD domain may be altered to directly promote BAX oligomerization on the mitochondrial outer membrane, outer-membrane permeabilization and cytochrome $c$ release, leading to cell death. Mitochondrial dysfunction and stresses such as starvation can trigger mitochondrial hyperfusion, which is dependent on maintenance of mitochondrial membrane potential (red) and the presence of both long and short OPA1 isoforms. Hyperfused mitochondria can transiently buffer the effects of respiratory chain dysfunction and do not enter the mitophagy pathway. The relationship between UPRmt and mitochondrial shape has not been explored. 